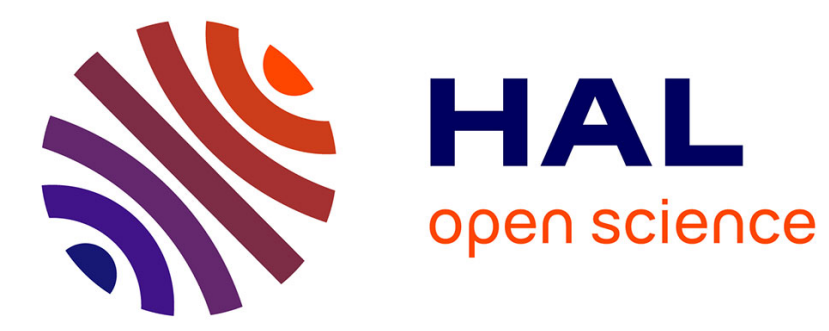

\title{
Confinement of dilute solutions of living polymers
}

\author{
V. Schmitt, F. Lequeux, C. Marques
}

\section{To cite this version:}

V. Schmitt, F. Lequeux, C. Marques. Confinement of dilute solutions of living polymers. Journal de Physique II, 1993, 3 (6), pp.891-902. 10.1051/jp2:1993173 . jpa-00247877

\section{HAL Id: jpa-00247877 https://hal.science/jpa-00247877}

Submitted on 1 Jan 1993

HAL is a multi-disciplinary open access archive for the deposit and dissemination of scientific research documents, whether they are published or not. The documents may come from teaching and research institutions in France or abroad, or from public or private research centers.
L'archive ouverte pluridisciplinaire HAL, est destinée au dépôt et à la diffusion de documents scientifiques de niveau recherche, publiés ou non, émanant des établissements d'enseignement et de recherche français ou étrangers, des laboratoires publics ou privés. 
Classification

Physics Abstracts

$36.20-61.25 \mathrm{H}-68.15$

\title{
Confinement of dilute solutions of living polymers
}

\author{
V. Schmitt ( $\left.{ }^{1}\right)$ F. Lequeux $\left({ }^{1}\right)$ and C. M. Marques $\left({ }^{2}\right)$ \\ (1) Laboratoire d'Ultrasons et de Dynamique des Fluides Complexes (*), Institut Le Bel, 4 rue \\ Blaise Pascal, 67070 Strasbourg Cedex, France \\ (2) Institut Charles Sadron $(* *), 6$ rue Boussingault, 67083 Strasbourg Cedex, France \\ (Received 13 January 1993, accepted in final form 18 February 1993)
}

\begin{abstract}
We investigate the thermodynamic properties of a dilute solution of living polymers confined between two solid repulsive walls. We consider both the case of rigid and of flexible macromolecules. When the confined system is taken to be at equilibrium with an external reservoir the living polymers behave similarly to a polydisperse solution of unbreakable chains. However for closed gaps the behaviour is quite different from a classical polymer solution because the wormlike micelles can adapt their intrinsic polydispersity in order to release the confinement constraint. In particular, for rigid living polymers this leads to a divergence of the average chain-length in the limit of strong confinement, as well as to a non-monotonic behaviour of the pressure acting on the walls.
\end{abstract}

\section{Introduction.}

Living polymers are linear aggregates which undergo reversible reactions. These labile macromolecules are known to form in surfactant solutions [1], where they are sometimes named vermicelli, but they also form in liquid sulphur [2] or liquid selenium [3]. The reversibility of the reactions allows the aggregates to exchange material, a given monomer sitting, at different times, on different host chains. As a consequence, mass polydispersity is not a quenched variable, it rather results from the conditions of thermodynamic equilibrium. A simple description of the polydispersity is provided by the minimization of the mean-field Flory free-energy density

$$
F=k_{\mathrm{B}} T \int_{0}^{\infty} \mathrm{d} L c(L)[\log c(L)+E]
$$

with respect to the chain-length distribution $c(L)$, under the constraint of monomer

$\left(^{*}\right)$ U.R.A. 851 CNRS.

(**) U.P.R. 022 CNRS. 
conservation

$$
\int_{0}^{\infty} \mathrm{d} L \operatorname{Lc}(L)=\Phi
$$

where $E$ is the energy cost of creating two chain caps, $\Phi$ is the monomer volume-fraction and all lengths are multiples of some lower cutoff length usually associated with the size of one monomer (or with the diameter of the vermicelli). The chain-length distribution that minimizes equation (1) has the exponential form

$$
c_{0}(L)=\frac{\Phi}{\bar{L}^{2}} \mathrm{e}^{-L i \bar{L}}
$$

with an average length $\bar{L}$, which is a function of the monomer volume fraction and of the endcap energy :

$$
\bar{L}=\Phi^{1 / 2} \mathrm{e}^{(E+1) / 2}
$$

Interestingly, this result does not depend on the stiffness of the chains, it applies both to flexible and rigid vermicelli.

Because the contour length of the molecules can be very large [1] (for surfactant vermicelli the length can be tuned from $15 \mathrm{~nm}$ up to $1000 \mathrm{~nm}$ ) these systems are in practice the labile analogs of polydisperse macromolecular systems, and can be used for instance for their viscoelastic properties [1]. But polymers are also very important in interfacial phenomena, where they are widely used to control the stabilization of colloidal suspensions [4], to modify the wetting or adhesion properties of the surfaces [5], to stabilize foams and Newton black films [6], to tune the binding-to-unbinding transition in lyotropic smectic systems [7], etc. In any case the presence of the walls influences the polymers in two ways: it reduces the conformational entropy of the chain and it changes its enthalpy due to the particular affinity to the interface (repulsion or attraction) that the chain monomers might experience. The final interfacial configuration of the chain results from the balance between these two factors. Vermicelli posssess a third, unique mechanism of reaction to the presence of the walls : even in a close gap they can adapt their polydispersity in order to minimize the total free energy. In this paper we investigate systematically this effect for rigid and flexible vermicelli confined between two flat, repulsive walls. The gap where the chains are confined is taken to be either in equilibrium with a bulk reservoir or closed. The first geometry corresponds for instance to quasi-static experiments in a force machine, while the second is more relevant to thin films or transient measurements in a force machine when the migration time of the chains is much larger than the kinetic relaxation time. In each case we study the behaviour of the chains in the gap by monitoring the evolution of both the average chain length and of the force acting between the two plates, as a function of the distance between plates.

\section{Flexible polymers.}

2.1 Chains IN Equilibrium WiTh A RESERVoIR. - We first consider a dilute polydisperse solution of flexible polymers confined between two plates, separated by a distance $D$ and in equilibrium with an external polymer solution which acts as a chain reservoir. Under these conditions, the chain length distribution in the gap $c_{\mathrm{g}}$, is obtained by minimization of the grand potential density :

$$
\frac{\Omega_{\mathrm{g}}}{k_{\mathrm{B}} T}=\int_{0}^{\infty} c_{\mathrm{g}}(L, D)\left[\log c_{\mathrm{g}}(L, D)+E-\log Z_{\mathrm{D}}(L)-\mu_{0} L\right] \mathrm{d} L+\Pi_{\mathrm{ex}}
$$


where $\mu_{0}$ and $\Pi_{\mathrm{ex}}$ are respectively the monomer chemical potential and the osmotic pressure of the bulk solution in units of $k_{\mathrm{B}} T$. They can be calculated from equation (1) :

$$
\mu_{0}=\frac{\partial F}{\partial \Phi}=-\frac{1}{\bar{L}} \quad \Pi_{\mathrm{ex}}=\Phi \frac{\partial F}{\partial \Phi}-F=\frac{\Phi}{\bar{L}}
$$

The effect of confinement is accounted for by the factor $\log Z_{\mathrm{D}}(L)$, where $Z_{\mathrm{D}}(L)$ is the partition function of a Gaussian polymer chain confined between two impenetrable walls [8] :

$$
Z_{D}(L)=\frac{8}{\pi^{2}} \sum_{p \text { odd }} \frac{1}{p^{2}} \mathrm{e}^{-\frac{\pi^{2} L p^{2}}{6 D^{2}}}
$$

where $D$ is also taken as a multiple of the lower cutoff length associated with $L$.

For chains with a gyration radius $R=\sqrt{(L / 6)}$ much larger than the gap thickness $D$ we have a strong confinement and the partition function can be approximated by

$$
Z_{\mathrm{D}}=\frac{8}{\pi^{2}} \mathrm{e}^{-\frac{\pi^{2} L}{6 D^{2}}}
$$

In the reverse limit of weak confinement $(R \ll D)$, we have

$$
Z_{\mathrm{D}}=1-\frac{4}{\sqrt{\pi D}} R
$$

By minimizing the grand potential $\Omega$ with respect to $c_{\mathrm{g}}(L, D)$, we obtain a simple expression for the chain concentration inside the gap :

$$
c_{\mathrm{g}}(L, D)=Z_{\mathrm{D}}(L) c_{0}(L)
$$

where $c_{0}(L)$ is the chain bulk distribution (3). The length distribution of small chains $(R \ll D)$ is only slightly perturbed from the bulk value. However, very large chains $(R \gg D)$ are almost completely excluded from the gap. Although no analytical expression is available for the partition function $Z_{D}$, all the integrated quantities like the volume fraction $\Phi_{\mathrm{g}}$ of monomers

$$
\Phi_{\mathrm{g}}=\int_{0}^{\infty} \mathrm{d} L c_{\mathrm{g}}(L, D) L
$$

the average length

$$
\left\langle L_{\mathrm{g}}\right\rangle=\frac{\Phi_{\mathrm{g}}}{\int_{0}^{\infty} \mathrm{d} L c_{\mathrm{g}}(L, D)}
$$

or the pressure

$$
\Pi=-\frac{\partial(D \Omega)}{\partial D}
$$

can be exactly computed by performing a Laplace transform on $Z_{\mathrm{D}}(L)$ [9]. We get for the 
monomer volume fraction inside the gap :

$$
\Phi_{\mathrm{g}}=\Phi_{0}\left[1-\frac{3}{2} \frac{2}{X_{\mathrm{f}}} \tanh \left(\frac{X_{\mathrm{f}}}{2}\right)+\frac{1}{2 \cosh ^{2}\left(\frac{X_{\mathrm{f}}}{2}\right)}\right]
$$

where we have introduced the reduced variable $X_{\mathrm{f}}=D / R_{\mathrm{G}}, R_{\mathrm{G}}$ being the radius of gyration of a chain of unperturbed chain length $\bar{L}, R_{\mathrm{G}}=\sqrt{(\bar{L} / 6)}$.

In figure 1 we plot the partition coefficient $\alpha=\Phi_{\mathrm{g}} / \Phi_{0}$. For distances $D$ much larger than $R_{\mathrm{G}}$, the monomer volume fraction in the gap deviates only slightly from the bulk value. For a strong confinement $\left(D \ll R_{\mathrm{G}}\right.$ ), the partition coefficient vanishes like $\Phi_{\sigma} 30 X_{\mathrm{f}}^{4}$. It is important to stress that, due to the intrinsic polydispersity, the partition coefficient of the vermicelli does not vanish as fast as the partition coefficient of a monodisperse solution of chains of length $\bar{L}$. Indeed, for the monodisperse system, the partition coefficient vanishes exponentially as soon as the separation distance between plates is smaller than the radius of gyration [10]. The average chain length in the gap

$$
\left\langle L_{\mathrm{g}}\right\rangle=\frac{\bar{L}\left[1-\frac{3}{2} \frac{2}{X_{\mathrm{f}}} \tanh \frac{X_{\mathrm{f}}}{2}+\frac{1}{2 \cosh ^{2}\left(\frac{X_{\mathrm{f}}}{2}\right)}\right]}{1-\frac{2}{X_{\mathrm{f}}} \tanh \frac{X_{\mathrm{f}}}{2}}
$$

also vanishes algebraically, from the bulk value $\bar{L}$ (see Fig. 2).

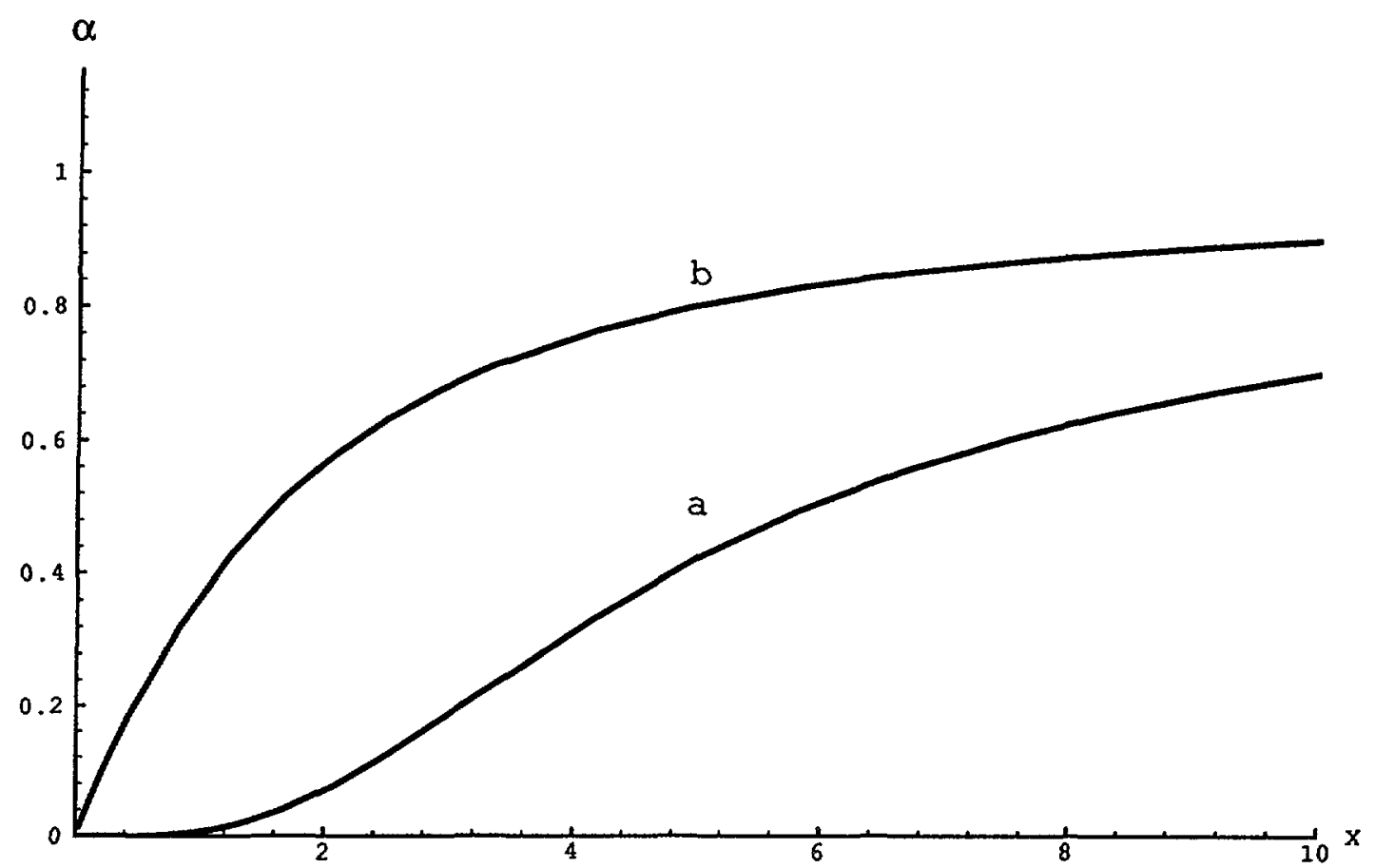

Fig. 1. - Dependence of the concentration partition coefficient $\alpha=\Phi_{\mathrm{g}} / \Phi_{0}$ for a living polymer dilute solution in an open gap : a) the case of flexible polymers $\left(X=D / R_{\mathrm{G}}\right)$; b) the case of rigid polymers $(X=D / \bar{L})$. 


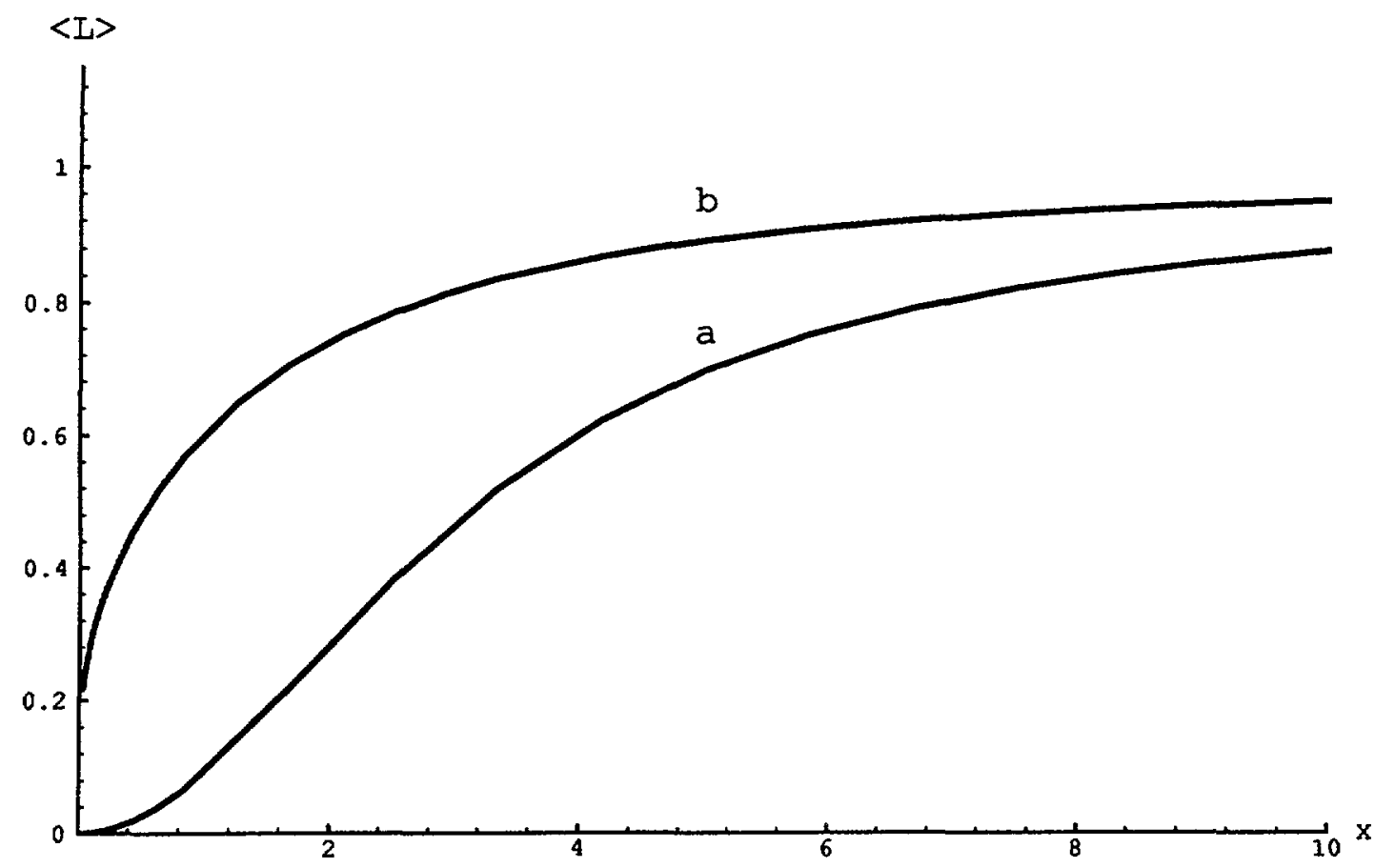

Fig. 2. - Dependence of the average chain-length of a dilute living polymer solution confined in an open gap : a) the flexible polymer case $\left(X=D / R_{\mathrm{G}}\right)$. For small values of $X$ the average chain-length behaves as $\left.X^{2} \bar{L} / 10 ; b\right)$ the case of rigid polymers $(X=D / \bar{L})$. For small values of $X$ the average chain-length behaves as $-1 /(\log X)$.

Because the chains are depleted from the surfaces, the force acting on the plates is attractive :

$$
\Pi=-\frac{\Phi_{0}}{\bar{L}}\left[\frac{1}{\cosh ^{2}\left(\frac{X_{\mathrm{f}}}{2}\right)}\right] .
$$

This effect is well known in colloidal systems where it leads to the so-called depletion inducedflocculation [11]. The pressure profile is plotted in figure 3. For vanishing small distances the curve has a parabolic shape $I \simeq-\Phi_{0} / \bar{L}\left(1-X_{\mathrm{f}}^{2} / 4\right)$.

2.2 RESTRICTED EQUILIBRIUM : THE CLOSED GAP. - We now consider a situation where the living polymers are confined in a closed gap. The volume and the monomer concentration are conserved for all separations $D$. This corresponds to a geometry of thin films deposited onto a solid surface or to a small drop confined between crossed cylinders in a force-machine. It is also relevant for experiments made with time scales much smaller than the diffusion time of the chains (as, for instance, a fast compression cycle in a force-machine). The free energy per unit volume now reads :

$$
F=\int_{0}^{\infty} \mathrm{d} L c(L, D)\left[\log c(L, D)+E-\log Z_{\mathrm{D}}(L)\right]
$$




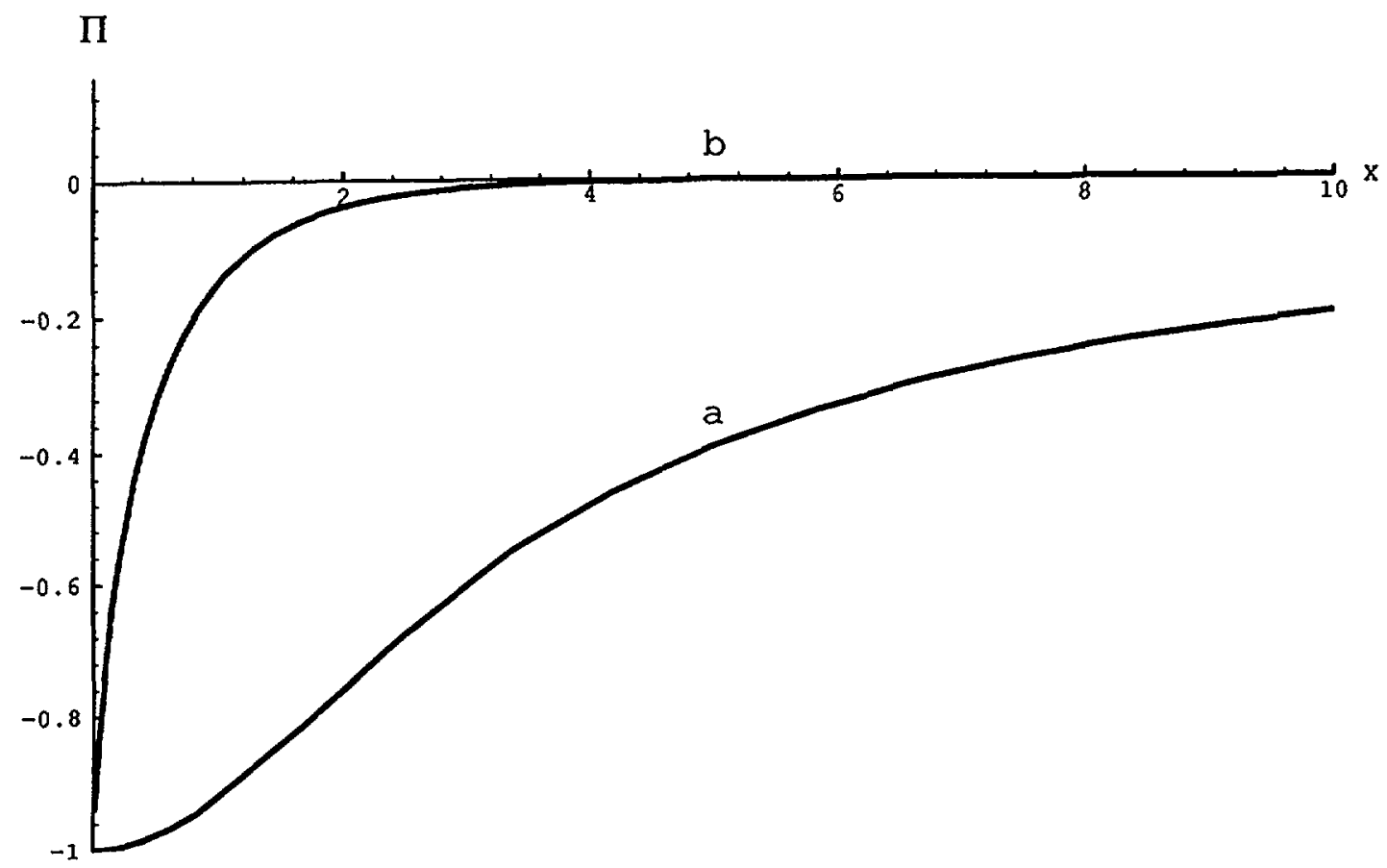

Fig. 3. - Dependence of the force per unit area acting on the plates for a dilute living polymer solution : a) the flexible polymer case $\left(X=D / R_{\mathrm{G}}\right)$; b) the rigid polymer case $(X=D / \bar{L})$.

Note that this expression is similar to equation (5) except for the chemical potential and the osmotic pressure terms which accounted therein for the equilibrium with the reservoir. The chain length distribution can be obtained in a similar manner by minimization of $F$ with respect to $c_{\mathrm{g}}(L, D)$, under the conservation condition (2). We get :

$$
c_{\mathrm{g}}(L, D)=\frac{\Phi_{0}}{\bar{L}^{2}} \mathrm{e}^{-L / \bar{L}_{\mathrm{D}}} Z_{\mathrm{D}}(L)
$$

where $1 / \bar{L}_{\mathrm{D}}$ is a Lagrange multiplier related to $\bar{L}$ by :

$$
\bar{L}^{2}=\bar{L}_{\mathrm{D}}^{2}\left[1-\frac{3}{2}\left(\frac{2}{X_{\mathrm{f}}} \tanh \frac{X_{\mathrm{f}}}{2}\right)+\frac{1}{2 \cosh ^{2} \frac{X_{\mathrm{f}}}{2}}\right]
$$

$X_{\mathrm{f}}$ is now a reduced variable associated with the Lagrange multiplier $1 / \bar{L}_{\mathrm{D}}$ and with the plate separation by $X_{\mathrm{f}}=D / \sqrt{\left(\bar{L}_{\mathrm{D}} / 6\right)}$.

Since the monomer volume fraction is conserved in the closed gap geometry, there are two quantities of interest : the average length in the gap and the pressure acting on the plates. The average chain length is given by:

$$
\langle L\rangle=\frac{\bar{L}^{2}}{\bar{L}_{\mathrm{D}}\left[1-\frac{2}{X_{\mathrm{f}}} \tanh \frac{X_{\mathrm{f}}}{2}\right]}
$$


a function plotted in figure 4. This length hardly varies from its unperturbed value $\bar{L}$ at infinite plate separation to a value $(\pi / \sqrt{8}) \bar{L}$ for vanishing small distances $D$. This surprising result can be understood as follows : for a strong confinement, the confinement energy is proportional to the number of chain segments $L, \log Z_{\mathrm{D}} \simeq L / D^{2}$. Thus this contribution to the free energy only changes the chemical potential per monomer, a quantity which adapts itself to satisfy the constraint of fixed volume fraction of monomers. On the other hand the equilibrium value of $c_{\mathrm{g}}(L, D)$ is only weakly perturbed by this contribution. Note that this result holds independently of the statistical nature of the chains, as long as strong confinement induces a penalty of $k_{\mathrm{B}} T$ per monomer. For chains with excluded volume, for instance, one has $\log Z_{\mathrm{D}}=L / D^{5 / 3}[12]$, only details of the curve shape in figure 4 would then be different. For instance the crossover distance is $D \sim \bar{L}^{3 / 5}$ instead of $D \sim \bar{L}^{1 / 2}$ and the multiplicative constant at the wall can be different as well. For a closed gap the pressure acting on the plates is positive :

$$
\Pi=-D \frac{\partial F}{\partial D}=\frac{\Phi}{\bar{L}} \frac{\bar{L}_{\mathrm{D}}}{\bar{L}}\left[\frac{2}{X_{\mathrm{f}}} \tanh \frac{X_{\mathrm{f}}}{2}-\frac{1}{\cosh ^{2}\left(\frac{X_{\mathrm{f}}}{2}\right)}\right] .
$$

The profile of the force (see Fig. 5) is similar to the profile arising from a monodisperse solution of chains of length $\bar{L}$;

$$
\begin{array}{ll}
\Pi \simeq \frac{2}{\sqrt{6}} \frac{\Phi}{\bar{L}} \frac{\sqrt{\bar{L}}}{D} ; \quad D \rightarrow \infty \\
\Pi \simeq \frac{\pi^{2}}{3} \frac{\Phi}{\bar{L}} \frac{\bar{L}}{D^{2}} ; \quad D \rightarrow 0 .
\end{array}
$$

$1 /<L>$

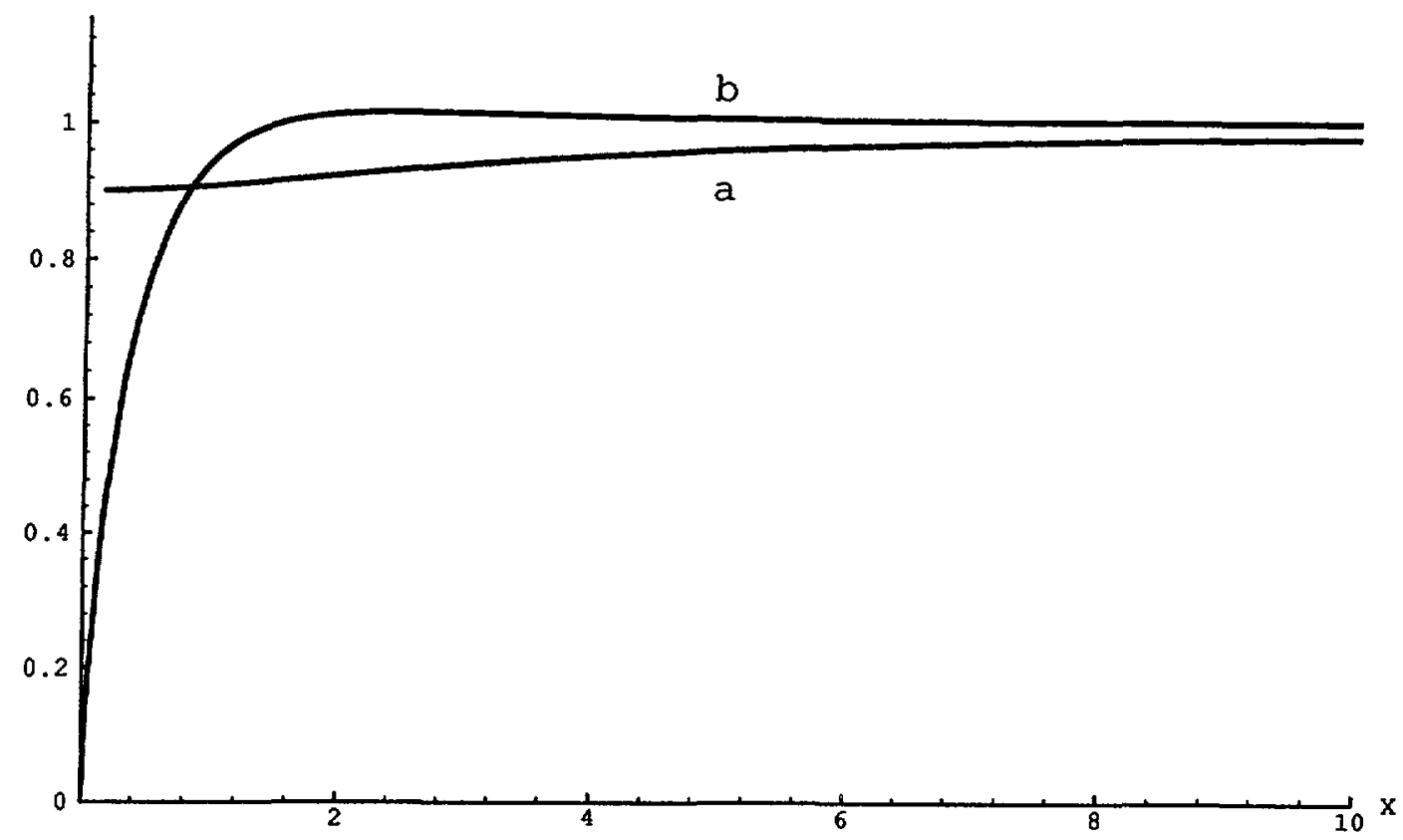

Fig. 4. - Dependence of the average number of living polymers in the closed gap geometry : a) flexible polymers $\left(X=D \sqrt{6} / \sqrt{\bar{L}_{\mathrm{D}}}\right) ;$ b) rigid polymers $\left(X=D / \bar{L}_{\mathrm{D}}\right)$. 


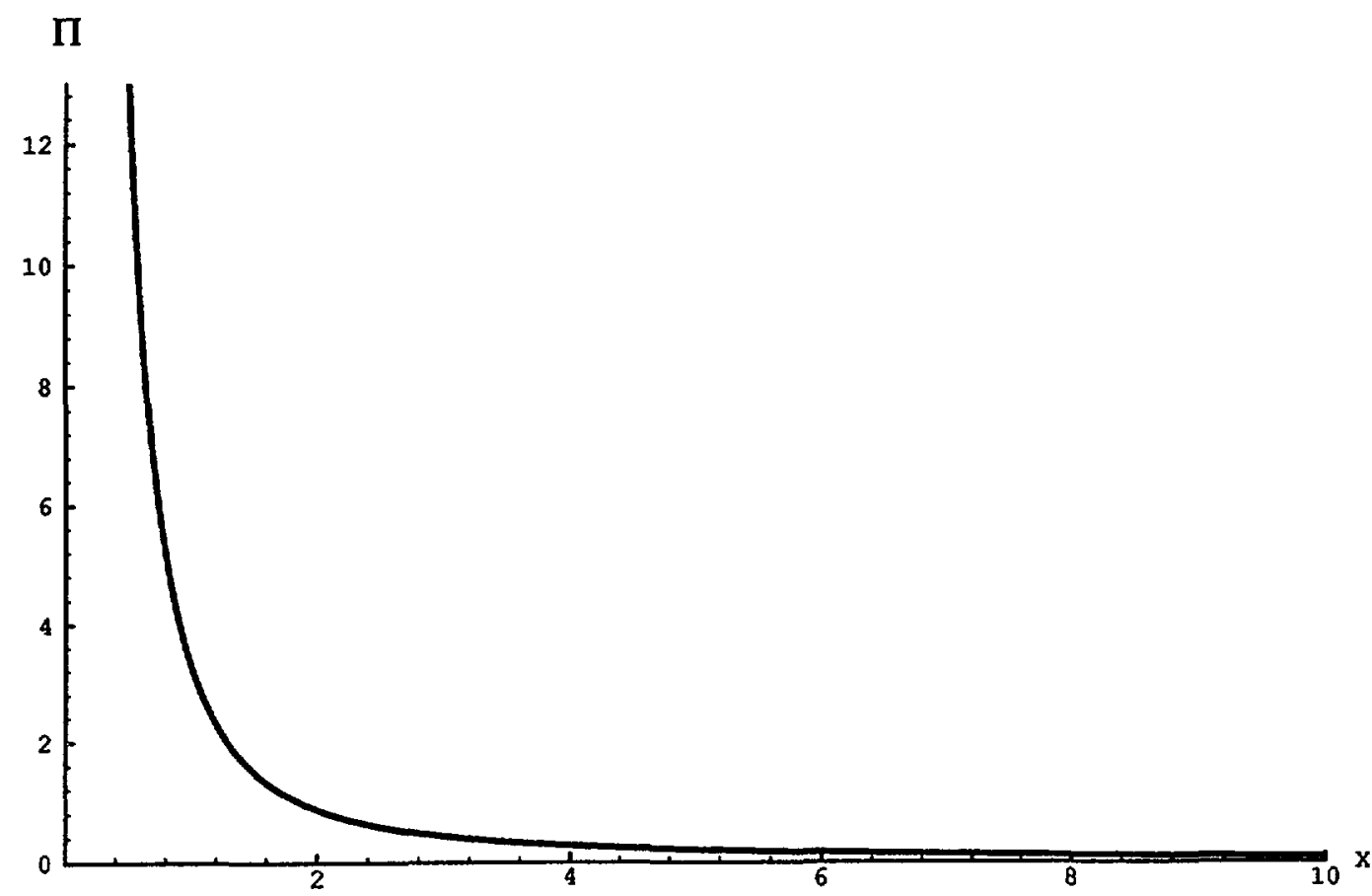

Fig. 5. - Dependence of the pressure acting on the plates for a dilute solution of flexible polymers confined in a closed gap $\left(X=D \sqrt{6} / \sqrt{\bar{L}_{D}}\right)$.

The asymptotic behaviour of the pressure can be simply obtained from the monodisperse results $(8,6)$ by remarking that the total pressure is simply the average of the pressure contribution from each given length $\langle\pi\rangle=\int \pi(L) c(L) \mathrm{d} L$.

\section{Rigid polymers.}

3.1 RIGID VERMICELLI IN AN OPEN GAP. - The limit of flexible polymers holds when the average contour length is much larger than the persistence length of the cylindrical aggregates. For a small monomer concentration or a low salt concentration, the persistence length can be large compared to the contour length of the polymers which behave then effectively as rigid objects. Rigid vermicelli have been recently studied, both experimentally [13] and theoretically $[14,15]$. Because of the intrinsic anisotropy of rod-like particles, one of the questions which often arises is the possibility of alignment of the molecules by an external field. It has been theoretically shown for instance [14], that, due to the positive feedback between alignment and growth, rod-like micelles in a flow undergo a « gelation » transition at a finite flow rate. In this section, we will study the effect of confining a dilute solution of rod-like vermicelli between two hard walls. When the solution is confined in an open gap, the grand-potential can be calculated from equation (5), with the partition function $Z_{\mathrm{D}}$ given by [16] :

$$
\begin{array}{lll}
Z_{\mathrm{D}}=1-\frac{L}{2 D} & \text { if } & D>L \\
Z_{\mathrm{D}}=\frac{D}{2 L} & \text { if } & D<L .
\end{array}
$$


For dilute solutions, the bulk chemical potential and the osmotic pressure do not depend on the rigidity of the chains, they are still given by equation (6). The concentration profile is obtained as before by minimization of the free energy with respect to the length distribution $c_{\mathrm{g}}(L, D)$ :

$$
\begin{array}{ll}
c_{\mathrm{g}}(L, D)=c_{0}(L)\left[1-\frac{L}{2 D}\right] & \text { if } \quad D>L \\
c_{\mathrm{g}}(L, D)=c_{0}(L) \frac{D}{2 L} & \text { if } \quad D<L .
\end{array}
$$

The monomer volume fraction and average length in the gap, as well as the pressure acting on the plates can then be straightforwardly calculated as in section 2.1 . We get the following expression for the monomer volume fraction inside the gap :

$$
\Phi_{\mathrm{g}}=\Phi_{0}\left[1+\frac{1}{X_{\mathrm{r}}}\left(\mathrm{e}^{-X_{\mathrm{r}}}-1\right)\right]
$$

where we have introduced the reduced variable $X_{\mathrm{r}}=D / \bar{L}$ (see Fig. 1).

The distance below which the volume fraction departs significantly from its bulk value $\Phi_{0}$ is the average rod length $\bar{L}$ (Fig. 2). Note also that, in contrast to the flexible polymer case, the volume fraction vanishes now linearly with the gap thickness $D$. The average rod length inside the gap is given by :

$$
\left\langle L_{\mathrm{g}}\right\rangle=\bar{L} \frac{1+\frac{1}{X_{\mathrm{r}}}\left(\mathrm{e}^{-X_{\mathrm{r}}}-1\right)}{\left(1-\frac{\mathrm{e}^{-X_{\mathrm{r}}}}{2}\right)+\frac{1}{2 X_{\mathrm{r}}}\left(\mathrm{e}^{-X_{\mathrm{r}}}-1\right)-\frac{X_{\mathrm{r}}}{2} \operatorname{Ei}\left(-X_{\mathrm{r}}\right)}
$$

where $\mathrm{Ei}$ is the exponential integral function. It also vanishes for small separation distances, but with a logarithmic singularity $\left\langle L_{\mathrm{g}}\right\rangle \sim-1 / \log X_{\mathrm{r}}$.

The depletion pressure acting on the plates is

$$
\Pi=-\frac{\partial(D \Omega)}{\partial D}=-\frac{\Phi_{0}}{\bar{L}}\left[\mathrm{e}^{-X_{\mathrm{r}}}+X_{\mathrm{r}} \operatorname{Ei}\left(-X_{\mathrm{r}}\right)\right]
$$

For a small gap thickness the force acting on the plates varies almost linearly with the distance $\Pi=-\Pi_{\mathrm{ex}}-X_{\mathrm{r}} \log X_{\mathrm{r}}$. It is worth noting that the distance at which the force departs significantly from the bulk (say where $\left.\Pi=\left(\Pi_{\infty}-\Pi_{\mathrm{ex}}\right) / 2\right)$ is smaller than in the flexible case (see Fig. 3). Qualitatively however, the behaviour of the confined rod solution is similar to the confined solution of flexible polymers : at a given plate separation $D$, only the chains of size smaller than $D$ remain in the gap.

3.2 THE CLOSED GAP. - The case where the rod molecules are confined in a closed gap can be treated as the confined flexible polymers, with the appropriate partition function $Z_{\mathrm{D}}$ given by (23). Minimization of the free-energy with respect to $c_{\mathrm{g}}(L, D)$ leads to :

$$
c_{\mathrm{g}}(L, D)=\frac{\Phi_{0}}{\bar{L}^{2}} \mathrm{e}^{-L / \bar{L}_{\mathrm{D}}} Z_{\mathrm{D}}(L)
$$


where $1 / \bar{L}_{\mathrm{D}}$ is a $D$-dependent Lagrange multiplier related to $\bar{L}$ and $D$ :

$$
\bar{L}^{2}=\bar{L}_{\mathrm{D}}^{2}\left[1+\frac{1}{X_{\mathrm{r}}}\left(\mathrm{e}^{-X_{\mathrm{r}}}-1\right)\right]
$$

where $X_{\mathrm{r}}=D / \vec{L}_{\mathrm{D}} \cdot \bar{L}_{\mathrm{D}}$ diverges as $2 \bar{L}^{2} / D$ for small values of the separation distance $D$. Interestingly the average length $\langle L\rangle$

$$
\langle L\rangle=\frac{\bar{L}^{2}}{\bar{L}_{\mathrm{D}}\left[1-\frac{\mathrm{e}^{-X_{\mathrm{r}}}}{2}+\frac{1}{2 X_{\mathrm{r}}}\left(\mathrm{e}^{-X_{\mathrm{r}}}-1\right)-\frac{X_{\mathrm{r}}}{2} \operatorname{Ei}\left(-X_{\mathrm{r}}\right)\right]}
$$

diverges for small distances as $\langle L\rangle \simeq-\bar{L}^{2} /[D \log (D / \bar{L})]$ (see Fig. 4).

Note that for the situation considered here where the total volume $V$ is conserved, the lateral dimensions of the film diverge only as $1 / D^{-1 / 2}$. It is thus in principle possible to « gel» the film, i.e. to obtain rod molecules of sizes comparable to the sample size, when $D \sim \bar{L}^{4} / V$. This situation contrasts strongly with the case of flexible molecules where the average size of the chains remains roughly constant during compression. The difference in this behaviour is due to the different penalties payed by flexible molecules or rod-like molecules under confinement. As explained above it costs about $k_{\mathrm{B}} T$ per monomer to strongly confine a flexible molecule. The rigid molecule, on the other hand, only pays $k_{\mathrm{B}} T$ per chain. Because the total chain number is not conserved there is no chemical potential associated with that quantity, which can then vary in order to minimize the constraint imposed by the confinement. Moreover, the surprising behaviour of $\bar{L}$, which decreases for $D>\bar{L}$ and increases for $D<\bar{L}$ is due to the competition between orientation entropy and chemical equilibrium. For large gaps, the long chains cannot rotate freely and disappear into small chains, but for small gaps all the chains are nearly similarly hampered by the walls, chemical equilibrium then takes over favoring a small concentration of end-caps, which induces the growth of the average size of the chains.

As for the flexible case the pressure acting on the plates

$$
\Pi=-D \frac{\partial F}{\partial D}=\frac{\Phi}{\bar{L}} \frac{\bar{L}_{\mathrm{D}}}{\bar{L}}\left[\frac{1}{2 X_{\mathrm{r}}}\left(1-\mathrm{e}^{-X_{\mathrm{r}}}\right)-\frac{\mathrm{e}^{-X_{\mathrm{r}}}}{2}-\frac{X_{\mathrm{r}}}{2} \operatorname{Ei}\left(-X_{\mathrm{r}}\right)\right]
$$

is repulsive. It has however a non-monotonic behaviour, as plotted in figure 6 . Note that this does not correspond to a thermodynamic instability since the curvature of the total free-energy is always positive.

\section{Conclusion.}

We have described the thermodynamic properties of confined dilute solutions of flexible or rod-like living polymers. The labile nature of the macromolecules allows for an adaptation of their polydispersity in order to release the constraint imposed by confinement. When the chains are confined in an open gap, the usual equilibration mechanism with the reservoir implies that the living polymers behave like an equivalent polydisperse solution of polymers of frozen polydispersity. In such a configuration the labile nature of the molecule does not show up as far as the static properties (chain length distribution, partition coefficient between the gap and the reservoir, pressure acting on the plates) are concerned. 


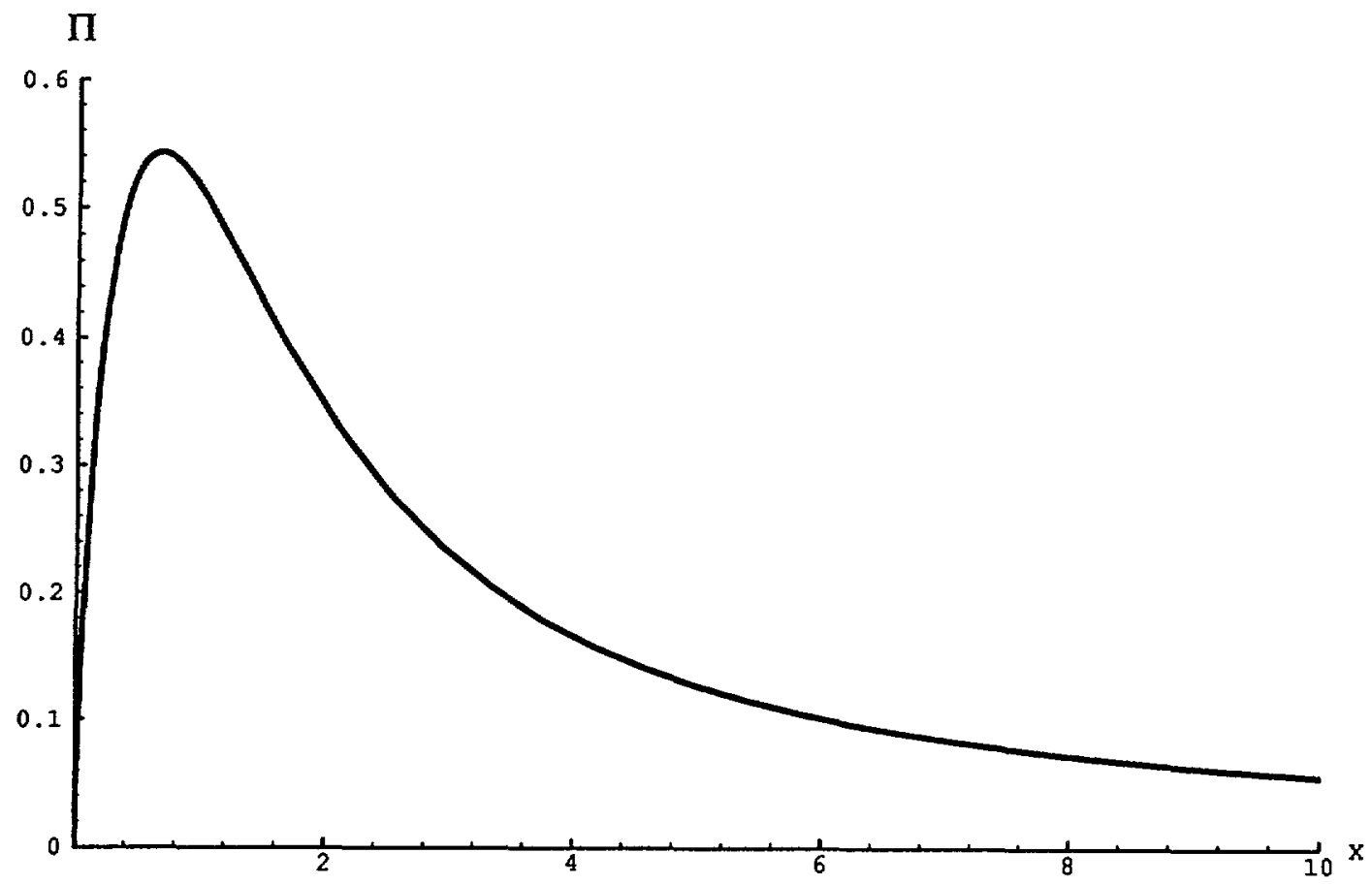

Fig. 6. - Dependence of the pressure acting on the plates for a dilute solution of rigid polymers in a closed gap $\left(X=D / \bar{L}_{\mathrm{D}}\right)$.

In the opposite situation where the vermicelli are confined in a closed gap, the relaxation of the confining constraint by adaptation of the chain length distribution plays a crucial role. However, for flexible chains, the induced modification of the polydispersity is not very large because the internal chemical potential associated with the conservation of the number of monomers can vary to almost compensate the effect of confinement. For rigid vermicelli this is not the case and a strong confinement induces a large growth in the average chain length which diverges as $-1 /[D \log (D / \bar{L})]$ for small distances. The chains could thus in principle reach dimensions comparable to the lateral dimensions of the thin film, leading to « gelation».

The confinement of semi-dilute living polymer solutions is also of great importance. We expect the thermodynamic equilibrium properties to be, in an open gap, identical to those of a polydisperse semi-dilute solution of classical polymers. In practice however the labile molecules present the advantage of releasing the entanglement constraints at a rate faster than the usual reptation rate in normal polymer solutions [1], and thus to equilibrate faster in a confining experiment. This allows for instance [17] the detection of depletion polymer forces in semi-dilute living polymer solutions, an otherwise very difficult task.

\section{Acknowledgments.}

We thank Dr. M. Cates and Dr. L. Auvray for very helpful discussions. Financial support from the British-French joint research program Alliance is gratefully acknowledged. 


\section{References}

[1] Cates M. E. and Candau S. J., J. Phys. Condens. Matter. 2 (1990) 6869 and references therein.

[2] Boue F., Ambroise J. P., Bellissent R., Pfeuty P., J. Phys. I France 2 (1992) 969.

[3] Faivre G., Gardissat J. L., Macromolecules 19 (1986) 1989.

[4] NAPPER D. H., Polymeric Stabilization of Colloidal Dispersions (Academic Press London, 1983).

[5] Johner A., Marques C. M., Phys. Rev. Lett. 69 (1992) 1827.

[6] Lionti-Addad S. and di Meglio J. M., Langmuir 8 (1992) 324.

[7] Brooks J. T., PhD thesis, Cambridge University (September 1992).

[8] DoI M. and Edwards S. F., The Theory of Polymer Dynamics (Oxford Science Publication, 1986).

[9] The infinite sums obtained by Laplace transformation can be found, for instance in « Table of Integrals, Series and Products » Gradshteyn and Ryzhik (Academic Press) p. 40.

[10] Russel W. B., Saville D. A., Schowalter W. R., Colloidal Dispersion (Cambridge University Press).

[11] Joanny J. F., Leibler L., De Gennes P. G., J. Pol. Sci. Polymer Phys. 17 (1979) 1073.

[12] DE Gennes P. G., Scaling Concepts in Polymer Physics (Cornell University Press).

[13] Rehage H. and Hoffmann H., J. Phys. Chem. 92 (1988) 4712.

[14] Turner M. S., Cates M. E., J. Phys. Condens. Matter. 4 (1992) 3719;

Wang S. Q., J. Phys. Chem. 94 (1990) 8381.

[15] Cates M. E., Marques C. M., Bouchaud J. P., J. Chem. Phys. 94 (1991) 8529.

[16] Auvray L., J. Phys. France 42 (1981) 79.

[17] RichetTI Ph., Private communication. 